\title{
Incidence and Survival of urothelial carcinoma of the urinary bladder in Norway $1981-2014$
}

\author{
B. K. Andreassen ${ }^{1 *}$, B. Aagnes ${ }^{4}$, R. Gislefoss', M. Andreassen ${ }^{2}$ and R. Wahlqvist ${ }^{3}$
}

\begin{abstract}
Background: Urothelial carcinoma of the urinary bladder (UCB) is the $4^{\text {th }}$ most common cancer type in men in developed countries, and tumor recurrence or progression occurs in more than half of the patients. Previous studies report contradictory trends in incidence and survival over the past decades. This article describes the trends of UCB incidence and survival from 1981 to 2014, including both invasive and non-invasive UCB using data from the Cancer Registry of Norway.

Methods: In Norway, 33,761 patients were diagnosed with UCB between 1981 and 2014. Incidence and 5-year relative survival were calculated, stratified by sex, morphology, stage, age and diagnostic period. Age-period-cohort models were used to distinguish period- and cohort effects. Temporal trends were summarized by calculating the average absolute annual change in incidence and relative survival allowing for breaks in this trend by incorporating a joinpoint analysis. Excess mortality rate ratios (EMRR) quantify the relative risks by using a proportional excess hazard model.
\end{abstract}

Results: The incidence of UCB in men increased from 18.5 (1981-85) to 21.1 (1991-95) per 100000 person-years and was rather stable thereafter (1996-2014). The incidence rates of UCB were lower in women increasing linearly from 4.7 to 6.2 over the past 34 years $\left(p=5.9 \cdot 10^{-7}\right)$. These trends could be explained by an increase of the incidence rates of non-invasive tumors. Furthermore, the observed pattern seemed to represent a birth cohort effect. Five-year relative survival increased annually with 0.004 in men $\left(p=1.3 \cdot 10^{-6}\right)$ and 0.003 in women $(p=4.5$. $10^{-6}$ ). There is a significant increase over the past 34 years in survival of UCB in both genders for local tumors but not for advanced stages.

Conclusions: Increasing and stable incidence trends mirror little improvement in primary and secondary prevention of UCB for more than three decades. Survival proportions increased only marginally. Thus, any changes in treatment and follow-up care did not lead to notable improvement with respect to survival of the patients. High estimates of preventable cases together with large recurrence rates of this particular cancer type, demand more research on prevention guidelines, diagnostic tools and treatment for UCB.

Keywords: Urothelial carcinoma of the urinary bladder, Bladder cancer, Incidence, Relative survival, Trends, Registry data, Epidemiology

\footnotetext{
* Correspondence: b.k.andreassen@kreftregisteret.no

${ }^{1}$ Department of Research, Cancer Registry of Norway, Institute for Population-based Research, Oslo, Norway

Full list of author information is available at the end of the article
} 


\section{Background}

Despite large global differences in the incidence of urothelial carcinoma of the urinary bladder (UCB), this cancer type remains the second most common genitourinary malignancy after prostate cancer in men worldwide. Globally, about 330000 new UCB cases were diagnosed and $123000 \mathrm{UCB}$ patients died from the disease in 2012. UCB is most frequent in Europe, Northern America, Western Asia and Northern Africa and least common in Eastern, Western and Middle Africa, Central America and the non-western regions of Asia [1]. Within Europe, UCB is the fourth most common cancer type in men with an age-standardized incidence rate (world) of 17.7 per 100000 person-years [1]. The corresponding rate in Norway is 20.8 per 100000 personyears based on the diagnosis period from 2009 to 2013 [2]. UCB incidence in women is much lower with 3.5 cases per 100000 person-years in Europe and 6.4 in Norway. International variation in UCB incidence have recently been described in detail [3], also including trends over time.

Several risk factors have been identified for UCB, tobacco smoking being the most predominant one. The population attributable risk for ever smoking has lately been estimated to be approximately $50 \%$ in both men and women in the US [4] and nearly $40 \%$ in the UK [5]. Men are more likely to get UCB with a male to female ratio of 3.2 in Norway [2]. UCB is also related to occupational exposure to certain chemicals like aromatic amines, chlorinated hydrocarbons and polycyclic aromatic hydrocarbons [6,7]. Patients previously irradiated for pelvic and abdominal malignancies are also at increased risk, as is also shown for intake of certain drugs used in previous cancer treatment [8] as well as diabetes medication [9]. UCB rarely occurs before the age of 40, and has a median age of diagnosis at 75 in the UK [10].

In Norway, $95 \%$ of all UCB cases are of the transitional cell type, and about $60 \%$ are primarily diagnosed without invasion into subepithelial connective tissue (tumor stage T1) or muscle (T2-4). These non-invasive tumors (papillary: Ta, Carcinoma in situ: Tis) and dysplasia are characterized by its high recurrence rates after transurethral resection of an initial tumor. Non-invasive papillary (Ta) tumors form the largest group and account for half of all diagnosed urothelial carcinomas. The majority of these tumors will recur, but the risk of progression to invasive UCB is low (4-7 \%) for lowgrade Ta tumors and approximately $12-23 \%$ for highgrade Ta tumors $[11,12]$. Carcinoma in situ seems to be more likely to progress than non-invasive papillary tumors, especially if concurrent with papillary tumors $[12,13]$. The high recurrence rates and the low but imminent progression risk leads to a tight follow-up of the patients with frequent visits and resource demanding treatment as well. This makes UCB one of the most expensive cancers to treat on a per-patient basis [14] in addition to being bothersome for the patients.

Incidence and survival trends for UCB in different countries based on cancer registry data have been described previously [15-17]. However, inclusion and exclusion criteria differ with respect to diagnose groups and periods as well as classification and registration practices. Furthermore, to our knowledge, none of the articles addressing UCB trends used a nationwide registry. We will describe the trends of UCB incidence and survival over time in Norway for all patients diagnosed with urothelial carcinoma of the urinary bladder between 1981 and 2014, including both invasive and noninvasive UCB (including dysplasia) using data from the Cancer Registry of Norway.

\section{Methods \\ Material}

The Cancer Registry of Norway has since 1953, compulsory by law, registered virtually all new cancer diagnoses in Norway. The registry receives information from three independent sources (clinicians, pathology laboratories, and from the Cause of Death Registry), which ensures completeness and high quality data [18]. Patients are identified through the unique national personal identification number assigned to all newborns and residents in Norway since 1960 . The present study comprises all new cases of histologically verified invasive and non-invasive urothelial carcinoma of the urinary bladder in the Norwegian population diagnosed between 1981 and 2014. UCB cases were selected based on morphological codes for the transitional cell type as presented in Table 1. UCB patients diagnosed before 1981 were excluded due to registration changes in the seventies mainly for non-invasive tumors. Thus, in total, 33,761 UCB patients were included in this study (Table 1). Participants were followed until death, migration or end of follow up on the $31^{\text {st }}$ of March 2016. The total follow-up time was 230783 person-years with a median follow-up time of 15.0 years. Out of all 33,761 UCB patients included in this study, 5228 individuals died from UCB according to the cause of death certificate.

Information on morphology, stage, grade, sex, age at diagnosis and date of diagnosis were retrieved from the Cancer Registry of Norway. Morphology, stage and grade were defined based on the most severe diagnosis within a 5 -month window including the first of the month when the first UCB diagnosis was received. We define five morphology groups based on the available tumor categories and grade information: Non-invasive papillary carcinoma low- and high-grade (Ta), non-invasive flat carcinoma (Tis), dysplasia (low-grade flat carcinoma) and 
Table 1 Patient inclusion criteria

\begin{tabular}{|c|c|c|c|c|}
\hline Morphology code & Description & Morphology group & Number of individuals & Percentage \\
\hline 8130 & Papillary, mild dysplasia, non-invasive (WHO-grade I) & Non-inv pap carcinoma LG & 6752 & $20.0 \%$ \\
\hline 8131 & Papillary, moderate dysplasia, non-invasive (WHO-grade II) & Non-inv pap carcinoma LG & 7644 & $22.6 \%$ \\
\hline 8136 & Papillary, not otherwise specified (NOS), non-invasive & Non-inv pap carcinoma LG & 238 & $0.7 \%$ \\
\hline 8132 & Papillary, non-invasive, high-grade (WHO-grade III) & Non-inv pap carcinoma HG & 2400 & $7.1 \%$ \\
\hline 8120 & Non-papillary, mild dysplasia, non-invasive (WHO-grade I) & Dysplasia & 303 & $0.9 \%$ \\
\hline 8121 & Non-papillary, moderate dysplasia, non-invasive (WHO-grade II) & Dysplasia & 589 & $1.7 \%$ \\
\hline 8126 & Non-papillary, NOS, non-invasive & Dysplasia & 441 & $1.3 \%$ \\
\hline 8122 & Non-papillary, non-invasive, high-grade (WHO-grade III) & Carcinoma in situ & 1198 & $3.5 \%$ \\
\hline 8123 & Non-papillary, NOS, invasive & Inv carcinoma & 319 & $0.9 \%$ \\
\hline 8124 & Non-papillary, mild dysplasia, invasive (WHO-grade I) & Inv carcinoma & 28 & $0.1 \%$ \\
\hline 8125 & Non-papillary,moderate dysplasia, invasive (WHO-grade II) & Inv carcinoma & 758 & $2.2 \%$ \\
\hline 8127 & Non-papillary, invasive, high-grade (WHO-grade III) & Inv carcinoma & 3471 & $10.3 \%$ \\
\hline 8133 & Papillary, NOS, invasive & Inv carcinoma & 169 & $0.5 \%$ \\
\hline 8134 & Papillary, mild dysplasia, invasive (WHO-grade I) & Inv carcinoma & 237 & $0.7 \%$ \\
\hline 8135 & Papillary, moderate dysplasia, invasive (WHO-grade II) & Inv carcinoma & 2205 & $6.5 \%$ \\
\hline 8137 & Papillary, invasive, high-grade (WHO-grade III) & Inv carcinoma & 3304 & $9.8 \%$ \\
\hline $80103^{\mathrm{a}}$ & Carcinoma in situ, NOS & Inv carcinoma & 185 & $0.5 \%$ \\
\hline $80203^{\mathrm{a}}$ & Carcinoma, undifferentiated, invasive, NOS & Inv carcinoma & 91 & $0.3 \%$ \\
\hline $812031^{\mathrm{a}}$ & Highly differentiated, invasive, G1, low-grade & Inv carcinoma & 84 & $0.2 \%$ \\
\hline $812032^{a}$ & Moderately differentiated, invasive, G2, low-grade & Inv carcinoma & 193 & $0.6 \%$ \\
\hline $812033^{\mathrm{a}}$ & Poorly differentiated, invasive, G3, high-grade & Inv carcinoma & 2180 & $6.5 \%$ \\
\hline $812034^{a}$ & Undifferentiated, invasive, G4, high-grade & Inv carcinoma & 27 & $0.1 \%$ \\
\hline \multirow[t]{2}{*}{$812039^{a}$} & NOS, invasive & Inv carcinoma & 945 & $2.8 \%$ \\
\hline & & Total number of individuals & 33761 & \\
\hline
\end{tabular}

Morphology codes (MOTNAC: Manual of Tumor Nomenclature and Coding from 1951 from the American Cancer Society), description, morphology groups and corresponding number and percentage of individuals included in this study

aCD-O: International Classification of Diseases for Oncology 1976-

invasive carcinoma (T1-T4). Grade information is based on WHO 1973 [19] and grouped into low (LG, WHO grade 1 and 2)- and high-grade (HG, WHO grade 3) (see Table 1). Stage is categorizsed as localized (non-invasive/ invasive cancer without any metastases), regional advanced (any infiltration into surrounding areas or regional metastases) and distant advanced (distant metastases) tumors. For the presentation of the results, age at diagnosis was divided into four age groups $(\leq 49,50-64,65-79$, $\geq 80$ ). The year of diagnosis is grouped into 5 -years intervals (diagnostic periods): 1981-1985, 1986-1990, ..., 2006-2010 and 2011-2014.

\section{Statistics}

Incidence rates (per 100000 person-years) were calculated based on the number of individuals getting their first UCB diagnosis and the number of individuals living in Norway for a certain sex, diagnostic time-period and age group. Age-specific incidence rates are presented for each of the four age-groups stratified for sex. Direct agestandardized incidence rates were calculated applying the World Standard Population [20] according to sex as well as age group, morphology group and stage across all diagnostic periods. Temporal trends of the incidence rates were best represented by a linear model, where the estimated regression coefficient $\hat{\beta}_{I}$ represents the average absolute annual incidence change. In order to summarize the observed trend over the last 34 years, this parameter has been provided together with the standard error and $p$-values for the test of an incidence change over time. We also implemented a joinpoint analysis [21] to uncover trends, which change over time. In order to interpret trends in age-specific incidence rates over time, we also apply an extension of the age-periodcohort model [22] to separate diagnosis period and birth cohort effects. An APC model incorporating restricted cubic splines, implemented in Stata was used [23, 24]. The APC model was applied for male patients with an 
age at diagnosis between 70 and 80, diagnosed between 1981 and 2010 and born between 1910 and 1930, which means that the model includes reliable information from the birth cohorts of interest.

5-year relative survival, based on the cohort approach, was estimated using the age-standardardized Ederer II method applying national population lifetables by sex, age group and diagnosis period [25]. The internal age-standardization used the age distribution of the last diagnostic period 2011-14 as weights. Temporal trends of 5 -year relative survival rates were best represented by a linear model. Thus, the average absolute change in annual 5-year relative survival estimates was provided together with the standard error and $p$-values for the test of a significant trend. We also implemented a joinpoint analysis [21] to uncover those trends, which change over time. The annual change in 5 -year survival proportions was estimated by the regression coefficient $\hat{\beta}_{R S}$. Because follow-up data for the latest diagnostic period are lacking, 5-year survival estimates for 2011-14 are based on a period approach. The corresponding column is marked with an $\left(^{*}\right)$ in order to emphasize the differently derived estimates and these estimates have not been included in the trend analysis.

We fitted a proportional excess hazard model [26-28] where sex, diagnostic period, age group, morphology group and stage were included as categorical variables. The baseline hazard was modelled using $5 \mathrm{df}$ for the spline variables using the Stata command stpm2 [29]. Excess mortality rates estimate the absolute difference between the expected mortality rate (from lifetables) and the observed mortality rate (from the data). The ratio of these quantities, the excess mortality rate ratios (EMRR), are in their interpretation similar to the hazard rate ratio and thus represent the factor of which patients under a certain condition are more likely to die compared to patients under another condition. EMRRs are reported together with $95 \%$ confidence intervals (CI).

All statistical analyses were performed in Stata 14/MP for Windows [30].

\section{Results}

\section{Overview}

A total number of 33,761 cases was diagnosed with UCB (transitional cell type) between 1981 and 2014. An overview over these patients with respect to sex as well as vital status (UCB and other cancer related death, alive, unknown), morphology group, stage, and age by sex and combined is provided in Table 2. Three quarters of the UCB patients diagnosed between 1981 and 2014 were men. For all patients (men and women combined), the median age at diagnosis was 72 years (inter-quartile range: $64-79$ years). The majority of patients (50.5\%)
Table 2 Patient characteristics

\begin{tabular}{|c|c|c|c|}
\hline & Men & Women & All \\
\hline$\%$ & 75.6 & 24.4 & 100.0 \\
\hline Age [25-75\% percentile] & 72 [64-79] & $73[65-81]$ & $72[64-79]$ \\
\hline \multicolumn{4}{|l|}{ Morphology } \\
\hline $\begin{array}{l}\text { Non-inv pap trans carcinoma } \\
\text { low-grade }\end{array}$ & 43.4 & 43.0 & 43.4 \\
\hline $\begin{array}{l}\text { Non-inv pap trans carcinoma } \\
\text { high-grade }\end{array}$ & 7.6 & 5.6 & 7.1 \\
\hline Displasia & 3.9 & 4.1 & 3.9 \\
\hline Carcinoma in situ & 3.7 & 3.1 & 3.6 \\
\hline Inv trans carcinoma & 41.3 & 44.3 & 42.0 \\
\hline \multicolumn{4}{|l|}{ Stage } \\
\hline Localised & 91.5 & 89.9 & 91.1 \\
\hline Regional advanced & 5.6 & 5.9 & 5.7 \\
\hline Distant advanced & 2.9 & 4.1 & 3.2 \\
\hline \multicolumn{4}{|l|}{ Death } \\
\hline Bladder cancer & 15.0 & 17.2 & 15.5 \\
\hline Other cancer & 22.1 & 21.4 & 22.0 \\
\hline Other than cancer & 30.2 & 26.2 & 29.1 \\
\hline Unknown & 2.7 & 2.6 & 2.8 \\
\hline Alive & 30.0 & 32.6 & 30.6 \\
\hline
\end{tabular}

Distribution of age, morphology group, stage and cause of death by sex and in total in the study population of patients diagnosed with urothelial carcinoma of the urinary bladder in Norway from 1981 to 2014

were diagnosed with non-invasive papillary carcinoma Ta (43.4 \% low-grade, $7.1 \%$ high-grade). Invasive transitional carcinoma (T1-T4) accounted for $42.0 \%$ of all UCB cases in this study and carcinoma in situ (3.6\%) as well as dysplasia (3.9 \%) are the least frequent morphology groups. Out of all patients diagnosed between 1981 and 2014, 66.6 \% had died by $31^{\text {st }}$ of March 2016, either due to UCB (15.5\%), cancer (not UCB) (22.0\%) or causes other than cancer $(29.1 \%)$. Women had relatively more invasive (44.3 vs $41.3 \%$ ) and more advanced (10.0 vs $8.5 \%$ ) tumors than men.

\section{Incidence}

Age-standardized incidence rates (World) are summarized in seven diagnostic periods in Table 3 . In addition, estimates for the average annual incidence change $\left(\hat{\beta}_{I}\right)$ are provided. These quantities estimate the average incidence trend throughout the study period and give an indicator for whether (and how much) incidence changed linearly over the past 34 years. For men, after a slightly increasing trend in the 1980s, UCB incidence has been stable throughout the remaining study period. A joinpoint analysis revealed an increasing trend of 0.34 ( $p=0.048$ ) from $1981-89$ and a stable trend from 1990 to $2014\left(\hat{\beta}_{I}=0.02, p=0.531\right)$. Thus, on average, 
Table $\mathbf{3}$ Incidence rates

\begin{tabular}{|c|c|c|c|c|c|c|c|c|c|c|}
\hline & & 1981-1985 & 1986-1990 & 1991-1995 & $1996-2000$ & 2001-2005 & 2006-2010 & 2011-2014 & $\beta_{l}(S E)$ & $p$-value \\
\hline \multirow[t]{2}{*}{ Men } & $n$ & 2972 & 3321 & 3628 & 3575 & 3928 & 4192 & 3915 & & \\
\hline & & 18.5 & 19.7 & 21.1 & 19.7 & 20.6 & 20.4 & 21.4 & $0.070(0.021)$ & $0.002^{a}$ \\
\hline \multirow[t]{2}{*}{ Women } & $n$ & 974 & 1038 & 1110 & 1150 & 1262 & 1380 & 1316 & & \\
\hline & & 4.7 & 4.9 & 5.2 & 5.1 & 5.3 & 5.7 & 6.2 & $0.043(0.007)$ & $5.9 \mathrm{E}-07^{\mathrm{a}}$ \\
\hline \multirow[t]{15}{*}{ Men } & Age (years) & & & & & & & & & \\
\hline & $0-49$ & 2.0 & 1.8 & 2.1 & 2.0 & 1.9 & 1.8 & 2.5 & $0.008(0.007)$ & 0.246 \\
\hline & $50-64$ & 46.2 & 49.0 & 50.4 & 40.3 & 42.4 & 42.6 & 44.4 & $-0.196(0.102)$ & 0.065 \\
\hline & $65-79$ & 148.4 & 156.0 & 171.0 & 169.1 & 175.4 & 176.1 & 173.1 & $0.899(0.186)$ & $3.2 \mathrm{E}-05^{\mathrm{a}}$ \\
\hline & $80+$ & 194.1 & 256.2 & 263.1 & 268.0 & 301.8 & 286.1 & 317.8 & $3.47(0.45)$ & 7.1E- $09^{\mathrm{a}}$ \\
\hline & Morphology & & & & & & & & & \\
\hline & Non-inv pap carcinoma LG & 8.7 & 9.5 & 9.9 & 9.2 & 9.5 & 8.9 & 8.3 & $-0.016(0.016)$ & 0.335 \\
\hline & Non-inv pap carcinoma HG & 0.6 & 0.9 & 1.3 & 0.9 & 1.4 & 2.3 & 2.6 & $0.061(0.006)$ & $1.5 \mathrm{E}-10^{\mathrm{a}}$ \\
\hline & Dysplasia & 0.6 & 0.7 & 1.0 & 0.9 & 0.6 & 0.6 & 1.4 & $0.011(0.006)$ & 0.070 \\
\hline & Carcinoma in situ & 0.4 & 0.7 & 0.7 & 0.9 & 0.9 & 0.9 & 0.7 & $0.013(0.003)$ & $0.002^{\mathrm{a}}$ \\
\hline & Inv carcinoma & 8.2 & 8.0 & 8.2 & 7.8 & 8.2 & 7.7 & 8.4 & $-0.001(0.010)$ & 0.985 \\
\hline & Stage & & & & & & & & & \\
\hline & Localised & 16.6 & 17.9 & 19.6 & 18.2 & 18.6 & 18.5 & 19.9 & $0.072(0.021)$ & $0.001^{\mathrm{a}}$ \\
\hline & Regional advanced & 1.3 & 1.1 & 1.0 & 0.8 & 1.3 & 1.4 & 1.0 & $0.001(0.005)$ & 0.919 \\
\hline & Distant advanced & 0.6 & 0.7 & 0.5 & 0.7 & 0.7 & 0.6 & 0.4 & $-0.003(0.003)$ & 0.380 \\
\hline \multirow[t]{15}{*}{ Women } & Age (years) & & & & & & & & & \\
\hline & $0-49$ & 0.7 & 0.7 & 0.8 & 0.7 & 0.6 & 0.7 & 0.8 & $0.001(0.004)$ & 0.739 \\
\hline & $50-64$ & 12.3 & 13.0 & 12.8 & 11.6 & 13.8 & 14.1 & 15.0 & $0.083(0.034)$ & $0.021^{a}$ \\
\hline & $65-79$ & 34.2 & 35.7 & 39.2 & 41.3 & 41.2 & 44.8 & 47.5 & $0.425(0.061)$ & $6.4 \mathrm{E}-08^{\mathrm{a}}$ \\
\hline & $80+$ & 57.0 & 52.9 & 50.8 & 52.3 & 61.2 & 61.4 & 76.3 & $0.519(0.167)$ & $0.004^{a}$ \\
\hline & Morphology & & & & & & & & & \\
\hline & Non-inv pap carcinoma LG & 2.1 & 2.4 & 2.4 & 2.5 & 2.8 & 2.8 & 2.6 & $0.022(0.005)$ & 1.0E-04 ${ }^{\mathrm{a}}$ \\
\hline & Non-inv pap carcinoma HG & 0.1 & 0.1 & 0.3 & 0.2 & 0.2 & 0.4 & 0.6 & $0.014(0.002)$ & $5.4 \mathrm{E}-08^{\mathrm{a}}$ \\
\hline & Dysplasia & 0.2 & 0.2 & 0.2 & 0.2 & 0.2 & 0.2 & 0.6 & $0.006(0.003)$ & $0.037^{\mathrm{a}}$ \\
\hline & Carcinoma in situ & 0.1 & 0.2 & 0.1 & 0.2 & 0.2 & 0.2 & 0.1 & $0.002(0.001)$ & 0.233 \\
\hline & Inv carcinoma & 2.3 & 2.0 & 2.2 & 1.9 & 2.0 & 2.1 & 2.3 & $-0.001(0.005)$ & 0.823 \\
\hline & Stage & & & & & & & & & \\
\hline & Localised & 4.2 & 4.4 & 4.7 & 4.5 & 4.7 & 5.0 & 5.7 & $0.040(0.008)$ & $8.6 \mathrm{E}-06^{\mathrm{a}}$ \\
\hline & Regional advanced & 0.4 & 0.3 & 0.2 & 0.3 & 0.4 & 0.4 & 0.3 & $0.001(0.001)$ & 0.611 \\
\hline & Distant advanced & 0.2 & 0.2 & 0.2 & 0.2 & 0.2 & 0.3 & 0.2 & $0.002(0.001)$ & 0.172 \\
\hline
\end{tabular}

Age-standardized incidence rates (applying the World Standard Population) by sex, morphology group and stage as well as age-specific incidence rates stratified for 5 -years interval of diagnosis from 1981 to 2014. Average annual incidence changes $\left(\hat{\beta}_{l}\right)$ are reported together with standard error (SE) and the $p$-value for the test of a significant incidence trend. Significant $p$-values (based on the $5 \%$ threshold) are marked with ${ }^{a}$

the incidence increased by $0.34\left(\hat{\beta}_{I}\right)$ within a year or 2.7 from 1981 to 1989 . The remaining 26 years of the study period, the incidence increased on average 0.02 per year or 0.5 from 1990 to 2014. The incidence of UCB in men was between 18.3 and 21.4 per 100000 person-years for all diagnostic periods during the study period. The incidence of UCB was lower in women with incidence rates increasing from 4.7 to 6.2 over the past 34 years. The corresponding trend was significant: $\hat{\beta}_{I}=0.043\left(p=5.9 \cdot 10^{-7}\right)$.

In men, age-specific incidence rates were increasing during the past 34 years, especially for the oldest patients $\left(\hat{\beta}_{I}=3.5, p=7.1 \cdot 10^{-9}\right)$ and those between 65 and $79\left(\hat{\beta}_{I}=\right.$ $\left.0.9, p=3.2 \cdot 10^{-5}\right)$. Similar age-dependent incidence trends could also be seen in women, although less pronounced probably due to the lower number of cases. This observed 
pattern was most likely mainly due to a birth cohort effect as illustrated in Fig. 1. The APC model confirmed the ageeffect (Fig. 1a) and suggested a larger birth cohort effect compared to the diagnosis period effect (Fig. 1b). The incidence rate ratio (IRR) was 1.22 (CI: 1.04-1.45) when comparing the 1930 birth cohort to the one from 1910. This particular birth cohort effect stayed the same when reducing the model to an age-cohort model (results not shown).

In men, there was a significant increase in high-grade non-invasive papillary carcinoma (Ta HG: $p=1.5 \cdot 10^{-10}$ ) over the study period. Age-standardized incidence rates in men have been rather stable over the whole observation period for invasive carcinoma $(p=0.985)$ and lowgrade non-invasive papillary carcinoma (Ta LG $p=$ 0.335). The incidence of both high- and low-grade noninvasive papillary carcinoma (Ta) was increasing in women (HG $p=5.4 \cdot 10^{-8}$, LG $p=1.0 \cdot 10^{-4}$ ). The corresponding increase of incidence rates for carcinoma in situ has been less pronounced in women $(p=0.233)$, possibly due to small numbers of cases. A joinpoint analysis reveals that the increase in incidence rates of high-grade non-invasive papillary carcinoma is rather small until 2001 (men: $p=0.055$ ) and 2003 (women: $p$ $=0.105$ ), but significant thereafter (men: $p=1.0 \cdot 10^{-8}$, women: men: $p=1.0 \cdot 10^{-8}$ ).

Both genders showed an increasing trend with respect to the incidence rates for localized cancers. This trend was more pronounced for women $\left(p=8.6 \cdot 10^{-6}\right)$ than men $(p=0.001)$. Cases with advanced stage were rather rare, such that the interpretation suffered from small number of cases in both men and women. Still, there is a decreasing tendency for distant advanced tumors in men $(p=0.380)$, while the corresponding tendency in women is increasing $(p=0.172)$.

\section{Relative Survival}

Table 4 presents the sex-specific 5-year relative survival across 5-year diagnosis intervals from 1981 to 2014 stratified for age and morphology group as well as stage. Furthermore, the average annual survival change $\left(\hat{\beta}_{R S}\right)$ estimates the average trend of the 5-year relative survival
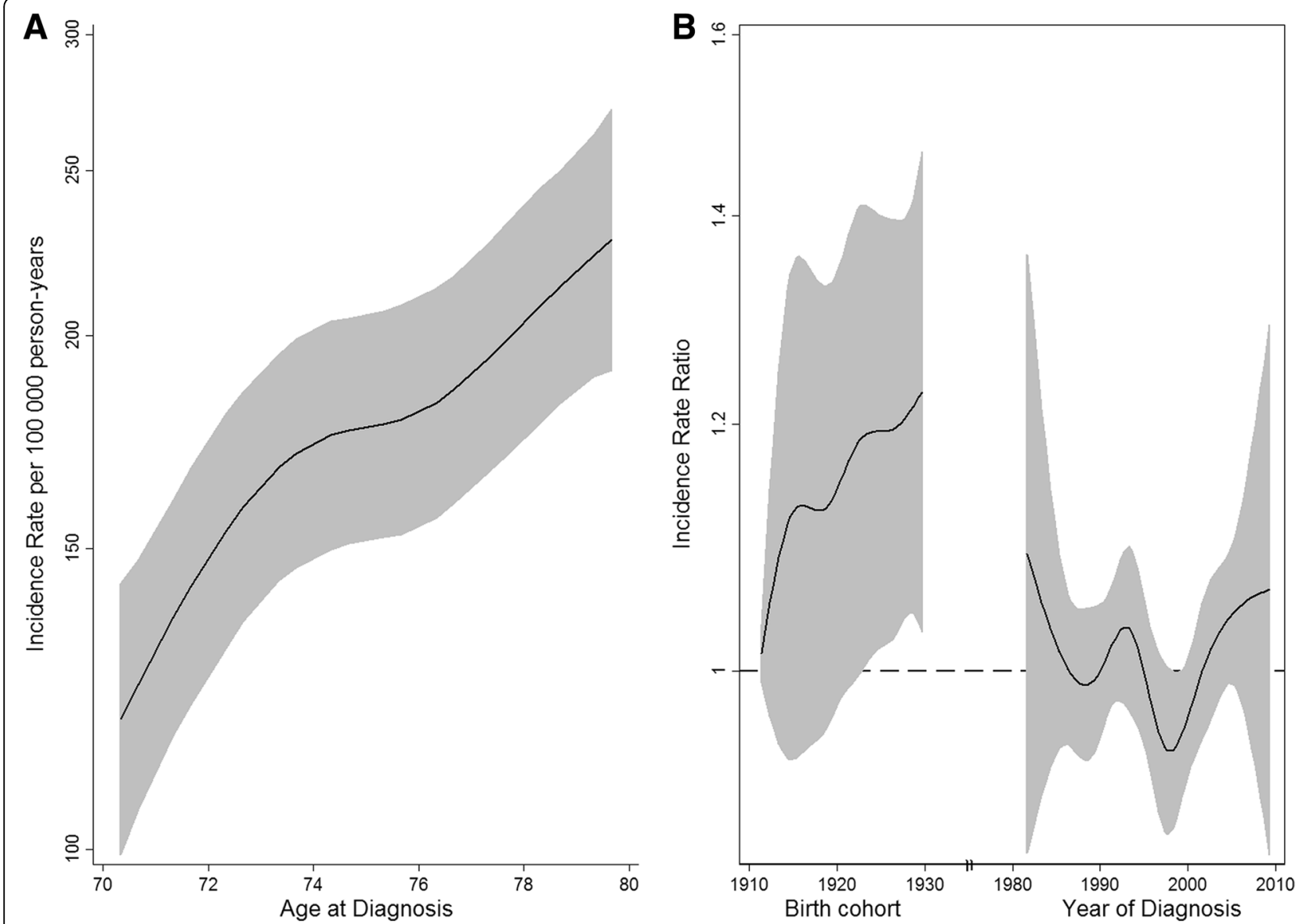

Fig. 1 Age-standardized incidence rates per 100000 person-years (a) and incidence rate ratios for birth cohort and diagnostic period effects for men diagnosed with urothelial carcinoma of the urinary bladder in Norway (b) 
Table 4 Relative survival proportions

\begin{tabular}{|c|c|c|c|c|c|c|c|c|c|c|c|c|c|c|c|c|c|}
\hline & \multirow[b]{2}{*}{ Men } & \multicolumn{2}{|c|}{$1981-85$} & \multicolumn{2}{|c|}{ 1986-90 } & \multicolumn{2}{|c|}{ 1991-95 } & \multicolumn{2}{|c|}{$1996-2000$} & \multicolumn{2}{|c|}{ 2001-05 } & \multicolumn{2}{|c|}{ 2006-10 } & \multicolumn{2}{|c|}{$2011-14^{\mathrm{a}}$} & \multirow{2}{*}{$\begin{array}{l}\beta_{R S}(S E) \\
0.004(0.001)\end{array}$} & \multirow{2}{*}{$\frac{p \text {-value }}{1.3 \mathrm{E}-06^{\mathrm{b}}}$} \\
\hline & & 0.67 & $(0.64,0.70)$ & 0.71 & $(0.68,0.74)$ & 0.73 & $(0.70,0.76)$ & 0.72 & $(0.70,0.75)$ & 0.75 & $(0.72,0.77)$ & 0.77 & $(0.75,0.79)$ & 0.77 & $(0.75,0.79)$ & & \\
\hline & Women & 0.63 & $(0.59,0.67)$ & 0.68 & $(0.64,0.72)$ & 0.65 & $(0.61,0.69)$ & 0.70 & $(0.67,0.74)$ & 0.70 & $(0.67,0.73)$ & 0.72 & $(0.69,0.75)$ & 0.74 & $(0.71,0.77)$ & $0.003(0.001)$ & $4.5 \mathrm{E}-06^{\mathrm{b}}$ \\
\hline \multirow[t]{15}{*}{ Men } & Age (years) & & & & & & & & & & & & & & & & \\
\hline & $0-49$ & 0.90 & $(0.84,0.95)$ & 0.85 & $(0.78,0.90)$ & 0.93 & $(0.88,0.97)$ & 0.92 & $(0.86,0.95)$ & 0.89 & $(0.83,0.93)$ & 0.91 & $(0.85,0.95)$ & 0.94 & $(0.84,0.98)$ & $0.001(0.001)$ & 0.623 \\
\hline & $50-64$ & 0.78 & $(0.74,0.81)$ & 0.81 & $(0.78,0.85)$ & 0.83 & $(0.80,0.86)$ & 0.84 & $(0.80,0.87)$ & 0.84 & $(0.81,0.86)$ & 0.83 & $(0.81,0.86)$ & 0.89 & $(0.85,0.91)$ & $0.003(0.001)$ & $0.006^{\mathrm{b}}$ \\
\hline & $65-79$ & 0.69 & $(0.65,0.72)$ & 0.68 & $(0.65,0.72)$ & 0.72 & $(0.70,0.75)$ & 0.73 & $(0.70,0.76)$ & 0.75 & $(0.72,0.78)$ & 0.78 & $(0.76,0.81)$ & 0.78 & $(0.73,0.81)$ & $0.005(0.001)$ & $7.6 \mathrm{E}-07^{\mathrm{b}}$ \\
\hline & $80+$ & 0.53 & $(0.45,0.63)$ & 0.65 & $(0.57,0.73)$ & 0.63 & $(0.56,0.71)$ & 0.60 & $(0.53,0.66)$ & 0.64 & $(0.58,0.70)$ & 0.68 & $(0.62,0.73)$ & 0.63 & $(0.54,0.72)$ & $0.007(0.003)$ & $0.015^{\mathrm{b}}$ \\
\hline & Morphology & & & & & & & & & & & & & & & & \\
\hline & Non-inv pap carcinoma LG & 0.85 & $(0.79,0.89)$ & 0.92 & $(0.87,0.95)$ & 0.91 & $(0.87,0.95)$ & 0.93 & $(0.88,0.96)$ & 0.91 & $(0.87,0.94)$ & 0.94 & $(0.90,0.97)$ & 0.93 & $(0.84,0.97)$ & $0.003(0.001)$ & $0.001^{b}$ \\
\hline & Non-inv pap carcinoma HG & 0.94 & $(0.18,1.00)$ & 0.76 & $(0.63,0.85)$ & 0.84 & $(0.71,0.92)$ & 0.80 & $(0.66,0.88)$ & 0.82 & $(0.72,0.88)$ & 0.87 & $(0.80,0.92)$ & 0.88 & $(0.77,0.94)$ & $0.004(0.002)$ & 0.050 \\
\hline & Dysplasia & 0.76 & $(0.56,0.87)$ & 0.63 & $(0.47,0.76)$ & 0.76 & $(0.61,0.86)$ & 0.83 & $(0.68,0.92)$ & 0.85 & $(0.67,0.93)$ & 0.83 & $(0.66,0.92)$ & 0.74 & $(0.54,0.86)$ & $0.004(0.003)$ & 0.155 \\
\hline & Carcinoma in situ & 0.79 & $(0.45,0.93)$ & 0.62 & $(0.48,0.74)$ & 0.64 & $(0.51,0.75)$ & 0.64 & $(0.53,0.73)$ & 0.84 & $(0.69,0.92)$ & 0.83 & $(0.71,0.90)$ & 0.91 & $(0.70,0.97)$ & $0.013(0.004)$ & $1.6 \mathrm{E}-04^{\mathrm{b}}$ \\
\hline & Inv carcinoma & 0.47 & $(0.43,0.51)$ & 0.49 & $(0.45,0.52)$ & 0.51 & $(0.48,0.55)$ & 0.50 & $(0.47,0.54)$ & 0.54 & $(0.50,0.57)$ & 0.55 & $(0.52,0.58)$ & 0.58 & $(0.54,0.63)$ & $0.003(0.001)$ & $2.3 \mathrm{E}-04^{\mathrm{b}}$ \\
\hline & Stage & & & & & & & & & & & & & & & & \\
\hline & Localised & 0.73 & $(0.69,0.76)$ & 0.76 & $(0.73,0.79)$ & 0.77 & $(0.74,0.79)$ & 0.77 & $(0.74,0.79)$ & 0.80 & $(0.78,0.82)$ & 0.82 & $(0.80,0.84)$ & 0.81 & $(0.77,0.84)$ & $0.004(0.001)$ & $7.0 \mathrm{E}-06^{\mathrm{b}}$ \\
\hline & Regional advanced & 0.25 & $(0.17,0.34)$ & 0.27 & $(0.20,0.36)$ & 0.28 & $(0.19,0.38)$ & 0.23 & $(0.15,0.31)$ & 0.25 & $(0.18,0.32)$ & 0.27 & $(0.21,0.33)$ & 0.22 & $(0.04,0.49)$ & $<0.001(0.002)$ & 0.991 \\
\hline & Distant advanced & 0.05 & $(0.02,0.11)$ & 0.08 & $(0.04,0.14)$ & 0.05 & $(0.01,0.15)$ & 0.04 & $(0.01,0.12)$ & 0.09 & $(0.04,0.17)$ & 0.07 & $(0.03,0.12)$ & 0.05 & $(0.01,0.030)$ & $-0.001(0.002)$ & 0.509 \\
\hline \multirow[t]{15}{*}{ Women } & Age (years) & & & & & & & & & & & & & & & & \\
\hline & $0-49$ & 0.90 & $(0.76,0.96)$ & 0.82 & $(0.68,0.90)$ & 0.87 & $(0.75,0.94)$ & 0.95 & $(0.84,0.99)$ & 0.82 & $(0.67,0.91)$ & 0.79 & $(0.65,0.88)$ & 0.78 & $(0.61,0.89)$ & $-0.003(0.003)$ & 0.409 \\
\hline & $50-64$ & 0.78 & $(0.71,0.84)$ & 0.85 & $(0.78,0.90)$ & 0.79 & $(0.72,0.84)$ & 0.84 & $(0.78,0.89)$ & 0.82 & $(0.76,0.86)$ & 0.89 & $(0.84,0.92)$ & 0.86 & $(0.77,0.92)$ & $0.004(0.002)$ & $0.032^{b}$ \\
\hline & $65-79$ & 0.61 & $(0.56,0.66)$ & 0.64 & $(0.59,0.69)$ & 0.65 & $(0.60,0.69)$ & 0.72 & $(0.67,0.76)$ & 0.74 & $(0.69,0.79)$ & 0.72 & $(0.67,0.76)$ & 0.70 & $(0.63,0.77)$ & $0.008(0.001)$ & $1.5 \mathrm{E}-05^{\mathrm{b}}$ \\
\hline & $80+$ & 0.51 & $(0.40,0.62)$ & 0.59 & $(0.48,0.70)$ & 0.52 & $(0.42,0.61)$ & 0.53 & $(0.44,0.62)$ & 0.51 & $(0.44,0.59)$ & 0.59 & $(0.51,0.66)$ & 0.68 & $(0.56,0.79)$ & $0.004(0.003)$ & 0.195 \\
\hline & Morphology & & & & & & & & & & & & & & & & \\
\hline & Non-inv pap carcinoma LG & 0.92 & $(0.82,0.96)$ & 0.94 & $(0.83,0.98)$ & 0.90 & $(0.82,0.94)$ & 0.92 & $(0.85,0.96)$ & 0.90 & $(0.84,0.94)$ & 0.91 & $(0.85,0.95)$ & 0.89 & $(0.78,0.95)$ & $-0.001(0.001)$ & 0.585 \\
\hline & Non-inv pap carcinoma HG & 0.74 & $(0.36,0.92)$ & 0.90 & $(0.34,0.99)$ & 0.71 & $(0.51,0.83)$ & 0.88 & $(0.27,0.99)$ & 0.79 & $(0.60,0.90)$ & 0.81 & $(0.68,0.89)$ & 0.88 & $((0.69,0.95)$ & $<0.001(0.005)$ & 0.986 \\
\hline & Dysplasia & 0.52 & $(0.35,0.66)$ & 0.65 & $(0.46,0.79)$ & 0.84 & $(0.46,0.96)$ & 0.78 & $(0.54,0.91)$ & 0.96 & $(0.11,1.00)$ & 0.78 & $(0.57,0.90)$ & 0.93 & $(0.46,0.99)$ & $0.011(0.005)$ & $0.035^{\mathrm{b}}$ \\
\hline & Carcinoma in situ & 0.39 & $(0.15,0.62)$ & 0.60 & $(0.41,0.75)$ & 0.73 & $(0.52,0.86)$ & 0.81 & $(0.57,0.93)$ & 0.62 & $(0.50,0.72)$ & 0.71 & $(0.51,0.84)$ & 0.83 & $(0.29,0.97)$ & $0.010(0.006)$ & 0.082 \\
\hline & Inv carcinoma & 0.43 & $(0.38,0.48)$ & 0.43 & $(0.37,0.48)$ & 0.42 & $(0.37,0.47)$ & 0.46 & $(0.41,0.51)$ & 0.45 & $(0.40,0.50)$ & 0.51 & $(0.46,0.56)$ & 0.50 & $(0.43,0.57)$ & $0.003(0.001)$ & $0.010^{\mathrm{b}}$ \\
\hline & Stage & & & & & & & & & & & & & & & & \\
\hline & Localised & 0.70 & $(0.66,0.74)$ & 0.74 & $(0.69,0.78)$ & 0.70 & $(0.66,0.73)$ & 0.76 & $(0.72,0.79)$ & 0.77 & $(0.74,0.80)$ & 0.79 & $(0.76,0.82)$ & 0.79 & $(0.74,0.83)$ & $0.004(0.001)$ & $9.2 \mathrm{E}-06^{\mathrm{b}}$ \\
\hline & Regional advanced & 0.14 & $(0.06,0.24)$ & 0.19 & $(0.10,0.29)$ & 0.18 & $(0.10,0.28)$ & 0.30 & $(0.20,0.40)$ & 0.18 & $(0.11,0.26)$ & 0.25 & $(0.16,0.35)$ & 0.30 & $(0.18,0.43)$ & $0.004(0.02)$ & 0.055 \\
\hline & Distant advanced & 0.04 & $(0.01,0.15)$ & 0.09 & $(0.01,0.27)$ & 0.02 & $(0.01,0.10)$ & 0.04 & $(0.01,0.10)$ & 0.06 & $(0.01,0.16)$ & 0.11 & $(0.05,0.22)$ & 0.08 & $(0.01,0.41)$ & $-0.010(0.006)$ & 0.136 \\
\hline
\end{tabular}

5 -year relative survival proportions (Ederer II) including confidence intervals by sex, morphology group and stage stratified by 5-year diagnosis intervals from 1981 to 2014 . We applied internal age-standardization based on the latest diagnostic period 2011-14. Average annual relative survival proportion changes $\left(\hat{\beta}_{R S}\right)$ are reported together with standard error (SE) and $p$-value for the test of a significant survival trend. ${ }^{a}$ The relative survival values for the latest diagnostic period are based on a period approach

Significant $p$-values (based on the $5 \%$ threshold) are marked with 
proportions throughout the study period. It gives an indicator for whether (and how much) survival changed over the past 30 years. On average, 5-year relative survival proportions increased annually by $\hat{\beta}_{R S}=0.004$ in men $(p=$ $\left.1.3 \cdot 10^{-6}\right)$ and $\hat{\beta}_{R S}=0.003$ in women $\left(p=4.5 \cdot 10^{-6}\right)$. In men, the 5 -year relative survival was 0.67 (CI: $0.64-0.70)$ in 1981-85 and 0.77 (CI: 0.75-0.79) in 2006-10. The corresponding 5-year relative survival in women were 0.63 (CI: 0.59-0.67) for 1981-85 and 0.72 (CI: 0.69-0.75) in 2006-10. The age-specific 5-year relative survival in men stayed mainly stable within the youngest age group ( $\leq 50$ years), while improvement in relative survival was the more pronounced the older the patient was ( $\geq 80$ years: $\hat{\beta}_{R S}=0.007$, $p=0.015)$. In women, these trends were similar except that the increase in 5-year relative survival was most distinct in the age group $65-79$ years $\left(\hat{\beta}_{R S}=0.008, p=1.5 \cdot 10^{-5}\right)$. There was a significant increase in 5 -year relative survival for invasive carcinomas in both men $\left(\hat{\beta}_{R S}=0.003, p=2.3 \cdot 10^{-4}\right)$ and women $\left(\hat{\beta}_{R S}=0.003, p=0.010\right)$. Relative survival for localized stages were significantly larger towards the end of the study period in both men $\left(\hat{\beta}_{R S}=0.004, p=7.0 \cdot 10^{-6}\right)$ and women $\left(\hat{\beta}_{R S}=0.004, p=9.2 \cdot 10^{-6}\right)$. There is no significant increase in survival for advanced stages, the tendency is towards decreasing survival for the distant advanced stage in both genders. Joinpoint analysis did not reveal any significant changes in the trend over time.

We estimated the influence from the variables considered in our study (sex, age, diagnosis period, morphology group, stage) on the relative survival estimates and the results are illustrated in Fig. 2. The largest impact on relative survival had a diagnosis where the patient had metastases and/or any infiltration into surrounding areas. Patients with distant advanced tumors are 8.5 times more likely to die than patients with localized tumors (excess mortality rate ratio $E M R R=8.5$, CI: 7.8-9.2). The corresponding EMRR for regional advanced tumors is 3.2 (CI: 3.0-3.4) when compared to localized tumors. The most severe diagnosis of having an invasive tumor lead to an EMRR of 6.1 (CI: 5.4-6.8) when comparing to the least severe diagnosis of a low-grade noninvasive papillary carcinoma. Carcinoma in situ (EMRR 3.2, CI: 2.7-3.9), dysplasia (EMRR 2.4, CI: 1.9-2.9) and highgrade papillary carcinoma (EMRR 2.3, CI: 1.9-2.7) also increased the EMRR significantly. The EMRR increased exponentially with age with an EMRR of 4.0 (CI: 3.3-4.8) for patients 80 years old (and older). The excess mortality rate was 1.2 times higher in women compared to men (CI: 1.11.3). All these effects were strongly significant.

\section{Discussion}

This paper presents trends in incidence and survival for patients diagnosed with urothelial carcinoma of the urinary bladder in Norway between 1981 and 2014.

Incidence of UCB in men and women has significantly increased throughout the study period. While this trend was rather linear for women, for men an initial increase of incidence rates in the eighties was observed, followed by stable incidence rates. Interestingly, a similar pattern has been observed in lung cancer, which is also a smoking-related cancer type. A recent report [31] showed that the lung cancer incidence rates in men levelled off while incidence rates in females were still

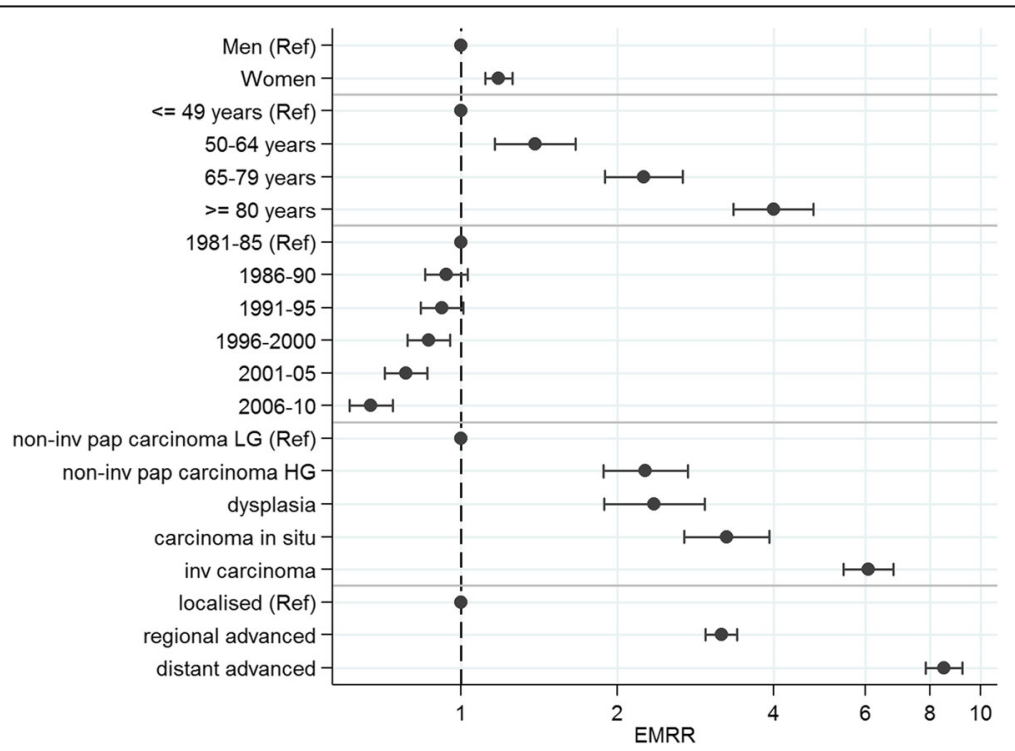

Fig. 2 Estimated excess mortality rate ratios (EMRRs) and confidence intervals for sex, age group, diagnostic period, morphology and stage based on a proportional excess hazard model 
increasing in Norway. A possible explanation is that the trends for lung cancer and UCB both reflect the variation in smoking habits in the Norwegian population. While the percentage of daily male smokers between 16 and 74 years has been almost linearly decreasing from 1973 (52 \%) to 2014 (13\%) in Norway, the corresponding percentage for female smokers kept stable from 1973 through 1999 (31-34 \%) and followed the smoking trend in men thereafter [32]. In addition, the application of an age-period-cohort model indicated that birth cohort effects, which are tightly connected to smoking patterns in the population, could at least partly, explain the observed trends. Furthermore, the overall increase in the incidence of UCB can be explained by a significant increase in the incidence of non-invasive tumours. In comparison to our results, Abdollah et al [16] observed a linear increasing trend in age-standardized incidence of UCB from 21.0 to 25.5 per 100000 person-years within the last three decades in US men. The same authors also reported an increased incidence for localized stages, which is in line with our observations. In South Australia, incidence increased during 1980 to 2004 for carcinoma in situ and invasive UCB combined [15] while invasive UCB stayed stable in Norway. However, we also observed an increase in high-grade non-invasive carcinoma (Tis, Ta) over time in men. Our data on sex differences in incidence are in line with other contemporary publications [33-35].

We have shown a small overall improvement in 5year relative survival proportions over the past three decades. This improvement can particularly be seen in older patients and in those suffering from carcinoma in situ. There is no significant survival improvement for advanced tumors in either sex. This could be interpreted as a sign of a negligible effect of the increased use of cystectomy during the study period. Since this treatment was also increasingly used for elderly patients, the same explanation could also apply to the small but apparent survival improvement in elderly patients. However, the largest improvement was seen for flat non-invasive tumors in women and noninvasive tumors in men, which might reflect the introduction of immunotherapy (Bacillus Calmette-Guerin) in the 1990s in Norway. In South Australia, there was a decrease in 5-year survival proportions from 64 to 58 \% observed during 1980 to 2004 for in situ and invasive UCB combined [15]. Survival trends in Switzerland for malignant UCB (non-invasive papillary and in situ carcinomas excluded) concluded with little survival improvement based on data from 1991 to 2010 [17]. A study investigating the variation of temporal trends with respect to sociodemographic and socioeconomic factors illustrated the underlying disparities associated with detection and treatment of
UCB and indicated the "necessity of debate of developing a valid screening procedure for UCB" at least for particular risk groups [16].

Modelling excess mortality with respect to the variables included in our study confirmed advanced tumors, invasive tumors, flat tumors and high-grade tumors as well as older age as risk factors. Sex and diagnosis period also play a significant role, but to a lesser extent. Sex differences in survival have been widely discussed. Differences with respect to the underlying anatomy, delays in diagnosis in females, as well as variations in hormone receptors and tumor biology might play a key role [36-38].

Relative survival estimates rely on population life tables rather than using cause-specific death information. Pros and cons have been widely discussed with a general agreement on the use of relative survival to estimate net survival for population-based studies. The main concern about using relative survival is the estimation of the underlying expected survival in the population. The latter needs to be a comparable group from the general population, comparable also with respect to certain confounders like smoking. The use of a general population lifetable, and not a lifetable adjusted for smoking, may have led to an underestimate of relative survival [39-41]. In a recent comparison of methods for estimating net survival, the Ederer II method [25] seemed to be the best choice [42].

The main limitation of this study is that we were not able to distinguish between muscle-invasive (T2-4) and non-muscle invasive (T1) cancers because these tumors are condensed to the same morphology group in our cancer registry. However, we did have information about the existence of metastases with tumors classified into three stages: localized (non-invasive/invasive cancer without any metastasis), regional advanced (any infiltration into surrounding areas or regional metastases) and distant advanced (distant metastases) tumors. The localized group contains tumors without metastases but also included those with unrecognized metastases. The reason for that is a coding change in 1993 with respect to the latter group, which does not allow us to separate nometastasis patients from those with unknown spread. Another drawback is that we had to use grading information based on the WHO 1973 system, such that our definition of low- and high grade is partly different from the WHO/ISUP 2004 system since we define low-grade tumors to include WHO grade 1 and 2 and high-grade tumors WHO grade 3 .

\section{Conclusion}

This study gives a comprehensive overview over incidence and survival changes related to a diagnosis of urothelial carcinoma of the urinary bladder in Norway from 1981 to 2014. To our knowledge, this is the only 
nationwide investigation of incidence and survival worldwide. The main conclusion is, that survival proportions increased only marginally. Thus, any changes in treatment and follow-up care did not lead to notable improvement with respect to survival of the patients. This conclusion gets support from several other studies looking at these trends over time [15-17]. High estimates of preventive cases together with high recurrence and progression rates of this particular cancer type, demands more research on prevention guidelines, diagnostic tools and treatment for bladder cancer.

\section{Abbreviations \\ Cl: Confidence interval; EMRR: Excess mortality risk ratio; HG: High-grade; LG: Low-grade; UCB: Urothelial carcinoma of the urinary bladder}

\section{Acknowledgements}

We thank Grete Kjølberg and Christine Mellem from the Cancer Registry of Norway for their support with the morphological classification of the bladder cancer patients.

\section{Funding}

Not applicable.

\section{Availability of data and materials}

Raw data can be obtained from the Cancer Registry of Norway.

\section{Authors' contributions}

BKA conceived, coordinated and designed the study, performed the statistical analysis and drafted the manuscript. BA participated in the statistical analysis and interpretation of the data. RW, MA and RG participated in the design of the study. All authors participated in writing of the manuscript and revised it critically. All authors have read and approved the final version of the manuscript.

\section{Competing interests}

The authors declare that they have no competing interests.

\section{Consent for publication}

Not applicable since no individual person's data which can identify a person is included in this manuscript.

\section{Ethics approval and consent to participate}

The research presented in this paper is based on data from the Cancer Registry of Norway. Their use is regulated by the Cancer Registry Regulations and does not require any additional approval or consents.

\section{Author details}

${ }^{1}$ Department of Research, Cancer Registry of Norway, Institute for Population-based Research, Oslo, Norway. ${ }^{2}$ Department of Pathology, Vestre Viken Hospital Trust, Drammen, Norway. ${ }^{3}$ Department of Urology, Oslo University Hospital, Oslo, Norway. ${ }^{4}$ Department of Registration, Cancer Registry of Norway, Institute for Population-based Research, Oslo, Norway.

Received: 27 January 2016 Accepted: 5 October 2016 Published online: 13 October 2016

\section{References}

1. Ferlay J, Soerjomataram I, Dikshit R, Eser S, Mathers C, Rebelo M, et al. Cancer incidence and mortality worldwide: sources, methods and major patterns in GLOBOCAN 2012. Int J Cancer. 2015;136(5):E359-386. doi:10.1002/ijc.29210

2. Cancer Registry of Norway. Cancer in Norway 2014 - Cancer incidence, mortality, survival and prevalence in Norway. Oslo: Cancer Registry of Norway; 2015. https://www.kreftregisteret.no/globalassets/cancer-in-norway/ 2014/cin2014-special_issue.pdf.
3. Chavan S, Bray F, Lortet-Tieulent J, Goodman M, Jemal A. International variations in bladder cancer incidence and mortality. Eur Urol. 2014;66(1): 59-73. doi:10.1016/j.eururo.2013.10.001.

4. Freedman ND, Silverman DT, Hollenbeck AR, Schatzkin A, Abnet CC Association between smoking and risk of bladder cancer among men and women. JAMA. 2011;306(7):737-45. doi:10.1001/jama.2011.1142.

5. Parkin DM, Boyd L, Walker LC. 16. The fraction of cancer attributable to lifestyle and environmental factors in the UK in 2010. Br J Cancer. 2011;105 Suppl 2:S77-81. doi:10.1038/bjc.2011.489.

6. Colombel M, Soloway M, Hideyuki A, Böhle A, Palou J, Buckley R, et al. Epidemiology, staging, grading, and risk stratification of bladder cancer. Eur Urol Suppl. 2008;7:618-26.

7. Brown T, Slack R, Rushton L. Occupational cancer in Britain. Urinary tract cancers: bladder and kidney. Br J Cancer. 2012;107 Suppl 1:S76-84. doi:10.1038/bjc.2012.121.

8. Pedersen-Bjergaard J, Ersboll J, Hansen VL, Sorensen BL, Christoffersen K, Hou-Jensen K, et al. Carcinoma of the urinary bladder after treatment with cyclophosphamide for non-Hodgkin's lymphoma. N Engl J Med. 1988; 318(16):1028-32. doi:10.1056/nejm198804213181604.

9. Mamtani R, Haynes K, Bilker WB, Vaughn DJ, Strom BL, Glanz K, et al. Association between longer therapy with thiazolidinediones and risk of bladder cancer: a cohort study. J Natl Cancer Inst. 2012;104(18):1411-21. doi:10.1093/jnci/djs328.

10. Cancer Research UK. http://www.cancerresearchuk.org/health-professional/ cancer-statistics/statistics-by-cancer-type/bladder-cancer\#heading-One. Accessed 9 Nov 2015.

11. Boman $\mathrm{H}$, Hedelin $\mathrm{H}$, Holmang S. Urine tissue-polypeptide-specific antigen (TPS) as a marker for bladder cancer. Scand J Urol Nephrol. 2001;35(4):270-4.

12. Liedberg F, Hagberg $O$, Holmang S, Hosseini Aliabad A, Jancke G, Ljungberg $B$, et al. Local recurrence and progression of non-muscle-invasive bladder cancer in Sweden: a population-based follow-up study. Scand J Urol. 2015; 49(4):290-5. doi:10.3109/21681805.2014.1000963.

13. Althausen AF, Prout Jr GR, Daly JJ. Non-invasive papillary carcinoma of the bladder associated with carcinoma in situ. J Urol. 1976;116(5):575-80.

14. Svatek RS, Hollenbeck BK, Holmang S, Lee R, Kim SP, Stenzl A, et al. The economics of bladder cancer: costs and considerations of caring for this disease. Eur Urol. 2014;66(2):253-62. doi:10.1016/j.eururo.2014.01.006.

15. Luke C, Tracey E, Stapleton A, Roder D. Exploring contrary trends in bladder cancer incidence, mortality and survival: implications for research and cancer control. Intern Med J. 2010;40(5):357-62. doi:10.1111/j.1445-5994. 2009.01980.x.

16. Abdollah F, Gandaglia G, Thuret R, Schmitges J, Tian Z, Jeldres C, et al. Incidence, survival and mortality rates of stage-specific bladder cancer in United States: a trend analysis. Cancer Epidemiol. 2013;37(3):219-25. doi:10.1016/j.canep.2013.02.002.

17. Bouchardy Magnin C, Lorez M, Clough-Gorr K. Trends in bladder cancer survival in Switzerland. Bull Suisse Cancer. 2013:2:141-6.

18. Larsen IK, Smastuen M, Johannesen TB, Langmark F, Parkin DM, Bray F, et al. Data quality at the Cancer Registry of Norway: an overview of comparability, completeness, validity and timeliness. Eur J Cancer. 2009;45(7):1218-31. doi:10.1016/j.ejca.2008.10.037

19. Mostofi F, Sobin L, Tosoni I. Histological Typing of Urinary Bladder Tumours. International Histological Classification of Tumours, No 19. Geneva: World Health Organization; 1973.

20. Doll R, Payne P, Waterhouse J, editors. Cancer incidence in five continents. A Technical Report. Berlin Heidelberg: Springer; 1966.

21. Kim HJ, Fay MP, Feuer EJ, Midthune DN. Permutation tests for joinpoint regression with applications to cancer rates. Stat Med. 2000;19(3):335-51.

22. Carstensen B. Age-period-cohort models for the Lexis diagram. Stat Med. 2007;26(15):3018-45.

23. Rutherford MJ, Thompson JR, Lambert PC. Projecting cancer incidence using age-period-cohort models incorporating restricted cubic splines. Int J Biostat. 2012;8(1):33. doi:10.1515/1557-4679.1411.

24. Rutherford MJ, Lambert PC, Thompson JR. Age-period-cohort modeling. Stata J. 2010;10(4):606

25. Ederer $\mathrm{F}$, Heise $\mathrm{H}$. Methodological note. 1959.

26. Dickman PW, Sloggett A, Hills M, Hakulinen T. Regression models for relative survival. Stat Med. 2004;23(1):51-64. doi:10.1002/sim.1597.

27. Nelson CP, Lambert PC, Squire IB, Jones DR. Flexible parametric models for relative survival, with application in coronary heart disease. Stat Med. 2007; 26(30):5486-98. doi:10.1002/sim.3064. 
28. Rutherford MJ, Crowther MJ, Lambert PC. The use of restricted cubic splines to approximate complex hazard functions in the analysis of time-to-event data: a simulation study. J Stat Comput Simul. 2015;85(4):777-93.

29. Lambert PC, Royston P. Further development of flexible parametric models for survival analysis. Stata J. 2009;9(2):265.

30. StataCorp. Stata Statistical Software: Release 14. College Station: StataCorp LP; 2015. http://www.stata.com/support/faqs/resources/citing-softwaredocumentation-faqs/.

31. Grimsrud TK, Skaug HK, Larsen IK. Lung cancer - changes in incidence by gender, age and county of residence 1984-2013. Tidsskr Nor Laegeforen. 2015;135(20):1844-9. doi:10.4045/tidsskr.14.1424.

32. Statistics Norway. 2015 [17.08.2016]. Available from: www.ssb.no.

33. Lucca I, Fajkovic H, Klatte T. Sex steroids and gender differences in nonmuscle invasive bladder cancer. Curr Opin Urol. 2014;24(5):500-5. doi:10.1097/mou.0000000000000092.

34. Rahmani AH, Alzohairy M, Babiker AY, Khan AA, Aly SM, Rizvi MA. Implication of androgen receptor in urinary bladder cancer: a critical mini review. Int J Mol Epidemiol Genet. 2013;4(3):150-5.

35. Izumi K, Zheng Y, Hsu JW, Chang C, Miyamoto H. Androgen receptor signals regulate UDP-glucuronosyltransferases in the urinary bladder: a potential mechanism of androgen-induced bladder carcinogenesis. Mol Carcinog. 2013:52(2):94-102. doi:10.1002/mc.21833.

36. Ristau BT, Davies BJ. Disparity in bladder cancer outcomes: what's sex got to do with it? Cancer. 2014;120(4):461-3. doi:10.1002/cncr.28420.

37. Patel MI, Bang A, Gillett D, Cheluvappa R, Smith DP. Poor survival of females with bladder cancer is limited to those aged 70 years or over: a populationwide linkage study, New South Wales, Australia. Cancer Med. 2015;4(8): 1145-52. doi:10.1002/cam4.452

38. Wolff I, Brookman-May S, May M. Sex difference in presentation and outcomes of bladder cancer: biological reality or statistical fluke? Curr Opin Urol. 2015;25(5):418-26. doi:10.1097/mou.0000000000000198.

39. Hinchliffe SR, Rutherford MJ, Crowther MJ, Nelson CP, Lambert PC. Should relative survival be used with lung cancer data? Br J Cancer. 2012;106(11): 1854-9. doi:10.1038/bjc.2012.182.

40. Blakely T, Soeberg M, Carter K, Costilla R, Atkinson J, Sarfati D. Bias in relative survival methods when using incorrect life-tables: lung and bladder cancer by smoking status and ethnicity in New Zealand. Int J Cancer. 2012;131(6): E974-982. doi:10.1002/ijc.27531.

41. Ellis $L$, Coleman MP, Rachet B. The impact of life tables adjusted for smoking on the socio-economic difference in net survival for laryngeal and lung cancer. Br J Cancer. 2014;111(1):195-202. doi:10.1038/bjc.2014.217.

42. Lambert PC, Dickman PW, Rutherford MJ. Comparison of different approaches to estimating age standardized net survival. BMC Med Res Methodol. 2015;15:64. doi:10.1186/s12874-015-0057-3.

\section{Submit your next manuscript to BioMed Central and we will help you at every step:}

- We accept pre-submission inquiries

- Our selector tool helps you to find the most relevant journal

- We provide round the clock customer support

- Convenient online submission

- Thorough peer review

- Inclusion in PubMed and all major indexing services

- Maximum visibility for your research

Submit your manuscript at www.biomedcentral.com/submit
Biomed Central 